\title{
Variational Iteration Method for Analytical Solution of the Lane- Emden Type Equation with Singular Initial and Boundary Conditions
}

\author{
Muhammad Nadeem $^{1}$ and Hijaz Ahmad ${ }^{2, *}$ \\ ${ }^{1}$ School of Mathematical Sciences, Dalian University of Technology, Dalian 116024, China \\ ${ }^{2}$ Department of Basic Sciences, University of Engineering and Technology Peshawar, Pakistan \\ E-mail: hijaz555@gmail.com \\ *Corresponding author
}

\begin{abstract}
In this paper, a well-known equation used in astrophysics and mathematical physics called the Lane-Emden equation is to be solved by a variational iteration method. The main purpose of this approach is to solve the singular initial value problems and also boundary value problem of Lane-Emden type equations. This technique overcomes its singularity at origin rapidly. It gives the approximate and exact solution with easily computable terms. The approach is illustrated with some examples to show its reliability and compactness.
\end{abstract}

\section{Introduction}

Lane-Emden type equations have been the focal point of various publications because of their frequent appearance in astrophysics, mathematical physics, engineering, mathematical biology and other fields. Recently, many mathematicians and physicists have been greatly attracted towards the study of singular initial value problems (IVPs) in second order ordinary differential equations of Lane-Emden type problems.

One of these type equations is formulated as

Received: March 18, 2019; Accepted: April 30, 2019

2010 Mathematics Subject Classification: 34-XX, 34Bxx, 34B05.

Keywords and phrases: Lane-Emden problems, singular IVPs, singular BVPs, variational iteration method.

Copyright ( $) 2019$ Hijaz Ahmad and Muhammad Nadeem. This is an open access article distributed under the Creative Commons Attribution License, which permits unrestricted use, distribution, and reproduction in any medium, provided the original work is properly cited. 


$$
y^{\prime \prime}+\frac{2}{x} y^{\prime}+f(y)=0, \quad x>0,0<x \leq 1,
$$

under the initial conditions

$$
y(0)=A, \quad y^{\prime}(0)=B,
$$

where $A$ and $B$ are constants and $f(y)$ is a real-valued continuous function. This equation was used to model various phenomena such as the theory of stellar structure, the thermal behavior of a spherical cloud of gas, isothermal gas spheres and the theory of thermionic currents $[1,2,3,4]$.

While, another class of singular initial value problems of Lane-Emden type can also be given in the form

$$
y^{\prime \prime}+\frac{\beta}{x} y^{\prime}+f(x, y)=g(x), \quad x>0,0<x \leq 1,
$$

under the initial conditions (2), where $f(x, y)$ is a real-valued continuous function and $g(x) \in C[0,1]$. For $\beta=2, f(x)=1, g(x)=0$, (3) shows the standard Lane-Emden type equation. The numerical solution of the Lane-Emden equation (3) as well as other types of linear and nonlinear singular IVPs in quantum mechanics and astrophysics [5, 6], is numerically challenging because of the singularity behavior at the origin. Hasan and Zhu $[7,8]$ have solved such a singular initial value problem by the Taylor series and modified Adomian decomposition methods. Gupta and Sharma [9] have also used the Taylor series method to solve Lane-Emden and Emden-Fowler equations. The approximate analytical solutions to the Lane-Emden equations were presented by Shawagfeh [10] and Wazwaz [11, 12, 13] using the Adomian decomposition method (ADM) [14]. He [15] obtained an approximate analytical solution of the Lane-Emden equation by applying a variational approach which uses a semi inverse method. Laio [16] solved Lane-Emden type equations by applying a homotopy analysis method. Chowdhury and Hashim [17] gave the solutions of class of singular second-order IVPs of Lane-Emden type by using He's homotopy perturbation method. Ramos [18] presented a series approach to the Lane-Emden equation and gave the comparison with He's homotopy perturbation method. Recently, Dehghan and Shakeri [19] first applied an exponential transformation to the Lane-Emden equation to overcome the difficulty of a singular point at $x=0$ and solved the resulting nonsingular problem by the variational 
iteration method. Exact solutions of generalized Lane-Emden solutions of the first kind are investigated by Goenner and Havas [20]. But analytical solutions are more needed to understand physical better.

Recently, a lot of attention has been devoted to the study of VIM such as study about the variational iteration method to find out the numerical solution of Sine-Gordon [21], Fokker-Plank equation [28]. [22] use the variational iteration method for solving biharmonic equation, [23, 24, 25, 19, 26] investigate various models, singular and nonsingular, linear and nonlinear, and ODEs and PDEs as well.

Our aim in this work to apply the VIM for these two types of Emden-Fowler equations with initial values and boundary values. In particular, we will first examine the Lane-Emden initial value problem given by [27]

$$
y^{\prime \prime}+\frac{\beta}{x} y^{\prime}+f(x, y)=0, \quad x>0,0<x \leq 1,
$$

under the initial conditions

$$
y(0)=A, \quad y^{\prime}(0)=B .
$$

Next, we will discuss the Lane-Emden boundary value problem

$$
y^{\prime \prime}+\frac{\beta}{x} y^{\prime}+f(x, y)=0, \quad x>0,0<x \leq 1,
$$

under the initial conditions

$$
y(0)=a, \quad y(0)=b .
$$

It is seen that for $\beta=1$ and $\beta=2$, both (4) and (6) shows cylindrical and spherical models [27].

\section{VIM and Lagrange Multipliers}

In this section, we will discuss about the variational iteration method (VIM) and find out the distinct Lagrange multipliers $\lambda(\eta)$ for different values of $\beta$. Consider the following nonlinear differential equation

$$
L[y(x)]+N[y(x)]=g(x),
$$

where $L$ is a linear operator, $N$ being a non-linear operator and $g(x)$ is known as 
analytical function. According to VIM, we can construct the following correction functional.

$$
y_{n+1}(x)=y_{n}(x)+\int_{0}^{x} \lambda(\eta)\left[L y_{n}(\eta)+N \tilde{y}_{n}(\eta)-g(\eta)\right] d \eta,
$$

where $\lambda(\eta)$ is general Lagrange multiplier which can be identified optimally via variational theory. $y_{0}(x)$ is an initial approximation with possible unknowns. $\tilde{y}_{n}$ is considered as restricted variation i.e., $\delta \tilde{y}_{n}=0$. Since the Lagrange multiplier $\lambda(\eta)$ is a crucial and critical in method as it may be a constant or a function. It can be seen that or first priority is to find the value of Langrage multiplier $\lambda(\eta)$ initially, which can be identified via optimally.

Taking the variation $\delta$ on both sides, we get as,

$$
\begin{aligned}
\delta y_{n+1}(x)= & \delta y_{n}(x)\left(1-\lambda^{\prime}(x)+\frac{\beta}{x} \lambda(x)\right)+\delta \lambda(x) y_{n}^{\prime}(x) \\
& +\delta \int_{0}^{x} y_{n}(\eta)\left(\lambda^{\prime \prime}(\eta)-\beta \frac{\eta \lambda^{\prime}(\eta)-\lambda(\eta)}{\eta^{2}}\right) d \eta .
\end{aligned}
$$

This in turn gives the stationary conditions

$$
\begin{gathered}
\lambda(\eta=x)=0, \\
\left.\lambda^{\prime}\right|_{\eta=x}=1, \\
\lambda^{\prime}-\beta \frac{\eta \lambda^{\prime}-\lambda}{x^{2}}=0 .
\end{gathered}
$$

Here we will discuss the following three cases to find out the value of $\lambda(\eta)$,

Case I. If $\beta=1$, (13) becomes as,

$$
\lambda(\eta)=\eta \ln \left(\frac{\eta}{x}\right)
$$

which shows for cylindrical problems. 
Case II. If $\beta=2$, (13) becomes as,

$$
\lambda(\eta)=\frac{\eta(\eta-x)}{x}
$$

which shows for spherical problems.

Case III. If $\beta>2$, (13) becomes as,

$$
\lambda(\eta)=\frac{\eta\left(\eta^{\beta-1}-x^{\beta-1}\right)}{(\beta-1) x^{\beta-1}}
$$

which shows for general problems.

Furthermore, by the successive approximation upon $y_{n+1}(x), n \geq 0$ for the solution of $y_{n}(x)$, will be readily obtained by using the obtained values of Lagrange multiplier and by using selective function $y_{0}$. Consequently, the exact solution can be obtained by using $y=\lim _{n \rightarrow \infty} y_{n}$.

\section{Lane-Emden IVPs}

In this section, initial value model of Lane-Emden with singular behavior $(x=0)$ for $\beta=1,2$ and $>2$ will be presented. Three initial value problems are discussed here.

\subsection{Example 1}

Consider the Lane-Emden type problem (4) with $\beta=1$,

$$
y^{\prime \prime}+\frac{1}{x} y^{\prime}+4 y^{2}-8 y^{3}=0
$$

with initial conditions

$$
y(0)=1, \quad y^{\prime}(0)=0
$$

the correctional functional for (17) is,

$$
y_{n+1}(x)=y_{n}(x)+\int_{0}^{x} \lambda(\eta)\left(y_{n}^{\prime \prime}(\eta)+\frac{1}{\eta} y_{n}^{\prime}(\eta)+4 y_{n}^{2}(\eta)-8 y_{n}^{3}(\eta)\right) d \eta,
$$


where $\lambda(\eta)=\eta \ln \left(\frac{\eta}{x}\right)$ as defined in above (14), considering the given initial value, we can select $y_{0}(x)=1$.

Using this selection in (19), we obtain the following successive approximation.

$$
\begin{aligned}
& y_{0}(x)=1 \\
& y_{1}(x)=1+x^{2} \\
& y_{2}(x)=1+x^{2}+x^{4}+\frac{5 x^{4}}{9}+\frac{x^{8}}{8} \\
& y_{3}(x)=1+x^{2}+x^{4}+x^{6}+\frac{8 x^{8}}{9}+\frac{307 x^{10}}{450}+\cdots \\
& y_{4}(x)=1+x^{2}+x^{4}+x^{6}+x^{8}+\frac{221 x^{10}}{225}+\frac{1891 x^{12}}{2025}+\cdots \\
& y_{5}(x)=1+x^{2}+x^{4}+x^{6}+x^{8}+x^{10}+\frac{2021 x^{12}}{2025}+\frac{14047 x^{14}}{14175} \cdots
\end{aligned}
$$

Since noise term vanish in limit which gives the exact solution of (17),

$$
y(x)=\frac{1}{1-x^{2}} .
$$

\subsection{Example 2}

Consider the Lane-Emden type problem (4) with $\beta=2$,

$$
y^{\prime \prime}+\frac{2}{x} y^{\prime}+y=x^{5}+30 x^{3}
$$

with initial conditions

$$
y(0)=0, \quad y^{\prime}(0)=0,
$$

the correctional functional for (21) is,

$$
y_{n+1}(x)=y_{n}(x)+\int_{0}^{x} \lambda(\eta)\left(y_{n}^{\prime \prime}(\eta)+\frac{2}{\eta} y_{n}^{\prime}(\eta)+y_{n}(\eta)-\eta^{5}-30 \eta^{3}\right) d \eta
$$


where $\lambda(\eta)=\frac{\eta(\eta-x)}{x}$ as defined in above (15), considering the given initial value, we can select $y_{0}(x)=0$.

Using this selection in (23), we obtain the following successive approximation.

$$
\begin{aligned}
& y_{0}(x)=0 \\
& y_{1}(x)=x^{5}+\frac{x^{7}}{56} \\
& y_{2}(x)=x^{5}-\frac{x^{9}}{5040} \\
& y_{3}(x)=x^{5}+\frac{x^{11}}{665280} \\
& y_{4}(x)=x^{5}-\frac{x^{13}}{121080960} \\
& y_{5}(x)=x^{5}+\frac{x^{15}}{29059430400}
\end{aligned}
$$

Since noise term vanish in limit which gives the exact solution of (21)

$$
y(x)=x^{5} .
$$

\subsection{Example 3}

Consider the Lane-Emden type problem (4) with $\beta>2$,

$$
y^{\prime \prime}+\frac{3}{x} y^{\prime}+8\left(1-\frac{x^{2}}{2}\right) y=0
$$

with initial conditions

$$
y(0)=1, \quad y^{\prime}(0)=0,
$$

the correctional functional for (25) is,

$$
y_{n+1}(x)=y_{n}(x)+\int_{0}^{x} \lambda(\eta)\left(y_{n}^{\prime \prime}(\eta)+\frac{3}{\eta} y_{n}^{\prime}(\eta)+8\left(1-\frac{\eta^{2}}{2}\right) y(\eta)\right) d \eta
$$


where $\lambda(\eta)=\frac{\eta\left(\eta^{\beta-1}-x^{\beta-1}\right)}{(\beta-1) x^{\beta-1}}$ as defined in above (16), considering the given initial value, we can select $y_{0}(x)=1$.

Using this selection in (27), we obtain the following successive approximation.

$$
\begin{aligned}
& y_{0}(x)=1 \\
& y_{1}(x)=1-x^{2}+\frac{x^{4}}{6} \\
& y_{2}(x)=1-x^{2}+\frac{x^{4}}{2}-\frac{x^{6}}{9}+\frac{x^{8}}{120} \\
& y_{3}(x)=1-x^{2}+\frac{x^{4}}{2}-\frac{x^{6}}{6}+\frac{13 x^{8}}{360}-\frac{23 x^{10}}{5400}+\cdots \\
& y_{4}(x)=1-x^{2}+\frac{x^{4}}{2}-\frac{x^{6}}{6}+\frac{x^{8}}{24}-\frac{43 x^{10}}{5400}+\frac{241 x^{12}}{226800}+\cdots \\
& y_{5}(x)=1-x^{2}+\frac{x^{4}}{2}-\frac{x^{6}}{6}+\frac{x^{8}}{24}-\frac{x^{10}}{120}+\frac{311 x^{12}}{226800}-\frac{143 x^{14}}{793800} \ldots
\end{aligned}
$$

Since noise term vanish in limit which gives the exact solution of (25),

$$
y(x)=e^{-x^{2}}
$$

\section{Lane-Emden BVPs}

In this section, boundary value model of Lane-Emden with singular behavior $(x=0)$ for $\beta=1,2$ and $>2$ will be presented. Three boundary value problems are discussed here.

\subsection{Example 4}

Consider the Lane-Emden type problem (6) with $\beta=1$,

$$
y^{\prime \prime}+\frac{1}{x} y^{\prime}-y=4+9 x-x^{2}-x^{3}
$$


with conditions

$$
y(0)=0, \quad y(1)=2,
$$

the correctional functional for (29) is,

$$
y_{n+1}(x)=y_{n}(x)+\int_{0}^{x} \lambda(\eta)\left(y_{n}^{\prime \prime}(\eta)+\frac{1}{\eta} y_{n}^{\prime}(\eta)-y(\eta)-4-9 \eta+\eta^{2}+\eta^{3}\right) d \eta \text {, }
$$

where $\lambda(\eta)=\eta \ln \left(\frac{\eta}{x}\right)$ as defined in above (14), considering the given initial value, we can select $y_{0}(x)=f x$, where $\mathrm{f}$ being any arbitrary constant.

Using this selection in (31), we obtain the following successive approximation.

$$
\begin{aligned}
& y_{0}(x)=f x \\
& y_{1}(x)=x^{2}+x^{3}+\frac{f x^{3}}{9}-\frac{x^{4}}{16}-\frac{x^{5}}{25} \\
& y_{2}(x)=x^{2}+x^{3}+\frac{f x^{5}}{225}-\frac{x^{6}}{576}-\frac{x^{7}}{1225} \\
& y_{3}(x)=x^{2}+x^{3}+\frac{f x^{7}}{11025}-\frac{x^{8}}{36864}-\frac{x^{9}}{99225} \\
& y_{4}(x)=x^{2}+x^{3}+\frac{f x^{9}}{893025}-\frac{x^{10}}{3686400}-\frac{x^{11}}{12006225} \\
& y_{5}(x)=x^{2}+x^{3}+\frac{f x^{11}}{108056025}-\frac{x^{12}}{530841600}-\frac{x^{13}}{2029052025}
\end{aligned}
$$

Imposing the boundary condition $y(1)=2$ in all approximations $y_{0}, y_{1}, y_{2}, y_{3}$, $y_{4}, y_{5}, \ldots$ and solving for every value of $f$, we obtain the following sequences for values of $f$.

$$
2,0.9225,0.574298,0.41083,0.316629,0.25681, \ldots
$$

Sequence shows that it converges to zero. Substituting $f=0$ in (32), 


$$
\begin{aligned}
& y_{0}(x)=0 \\
& y_{1}(x)=x^{2}+x^{3}-\frac{x^{4}}{16}-\frac{x^{5}}{25} \\
& y_{2}(x)=x^{2}+x^{3}-\frac{x^{6}}{576}-\frac{x^{7}}{1225} \\
& y_{3}(x)=x^{2}+x^{3}-\frac{x^{8}}{36864}-\frac{x^{9}}{99225} \\
& y_{4}(x)=x^{2}+x^{3}-\frac{x^{10}}{3686400}-\frac{x^{11}}{12006225} \\
& y_{5}(x)=x^{2}+x^{3}-\frac{x^{12}}{530841600}-\frac{x^{13}}{2029052025} \\
& \vdots
\end{aligned}
$$

Since noise term vanish in limit which gives the exact solution of (29)

$$
y(x)=x^{2}+x^{3} .
$$

\subsection{Example 5}

Consider the Lane-Emden type problem (6) with $\beta=2$,

$$
y^{\prime \prime}+\frac{2}{x} y=2\left(2 x^{2}+3\right) y
$$

with conditions

$$
y(0)=1, \quad y(1)=e
$$

the correctional functional for (34) is,

$$
y_{n+1}(x)=y_{n}(x)+\int_{0}^{x} \lambda(\eta)\left(y_{n}^{\prime \prime}(\eta)+\frac{2}{\eta} y_{n}^{\prime}(\eta)-\left(4 \eta^{2}+6\right)\left(y_{n}(\eta)\right)\right) d \eta
$$

where $\lambda(\eta)=\frac{\eta(\eta-x)}{x}$ as defined in above (15), considering the given initial value, we can select $y_{0}(x)=1+g x$, where $g$ being any arbitrary constant. 
Using this selection in (36), we obtain the following successive approximation.

$$
\begin{aligned}
y_{0}(x)= & 1+g x \\
y_{1}(x)= & 1+x^{2}+\frac{g x^{3}}{2}+\frac{x^{4}}{5}+\frac{2 g x^{5}}{15} \\
y_{2}(x)= & 1+x^{2}+\frac{x^{4}}{2}+\frac{g x^{5}}{10}+\frac{13 x^{6}}{105}+\frac{g x^{7}}{20}+\cdots \\
y_{3}(x)= & 1+x^{2}+\frac{x^{4}}{2}+\frac{x^{6}}{6}+\frac{3 g x^{7}}{280}+\frac{4 x^{8}}{105}+\frac{7 g x^{9}}{900}+\cdots \\
y_{4}(x)= & 1+x^{2}+\frac{x^{4}}{2}+\frac{x^{6}}{6}+\frac{x^{8}}{24}+\frac{g x^{9}}{1400}+\frac{47 x^{10}}{5775}+\frac{47 g x^{11}}{69300}+\cdots \\
y_{5}(x)= & 1+x^{2}+\frac{x^{4}}{2}+\frac{x^{6}}{6}+\frac{x^{8}}{24}+\frac{x^{10}}{120}+\frac{g x^{11}}{30800}+\frac{2489 x^{12}}{1801800}+\frac{4 g x^{13}}{105105}+\cdots \\
y_{6}(x)= & 1+x^{2}+\frac{x^{4}}{2}+\frac{x^{6}}{6}+\frac{x^{8}}{24}+\frac{x^{10}}{120}+\frac{x^{12}}{720}+\frac{3 g x^{13}}{2802800}+\frac{12499 x^{14}}{63063000} \\
& +\frac{251 g x^{15}}{168168000}+\cdots \\
y_{7}(x)= & 1+x^{2}+\frac{x^{4}}{2}+\frac{x^{6}}{6}+\frac{x^{8}}{24}+\frac{x^{10}}{120}+\frac{x^{12}}{720}+\frac{x^{14}}{5040}+\frac{3 g x^{15}}{112112000} \\
& +\frac{3323 x^{16}}{134008875}+\frac{53 g x^{17}}{1225224000} \cdots
\end{aligned}
$$

Imposing the boundary condition $y(1)=e$ in all approximations $y_{0}, y_{1}, y_{2}, y_{3}$, $y_{4}, y_{5}, \ldots$ and solving for every value of $g$, we obtain the following sequences for values of $g$.

$1.71828,0.81834,0.53462,0.398239,0.318091,0.26522,0.227647,0.199529, \ldots$.

Sequence shows that it converges to zero. Substituting $g=0$ in (37),

$$
\begin{aligned}
& y_{0}(x)=1 \\
& y_{1}(x)=1+x^{2}+\frac{x^{4}}{5}
\end{aligned}
$$




$$
\begin{aligned}
& y_{2}(x)=1+x^{2}+\frac{x^{4}}{2}+\frac{13 x^{6}}{105}+\cdots \\
& y_{3}(x)=1+x^{2}+\frac{x^{4}}{2}+\frac{x^{6}}{6}+\frac{4 x^{8}}{105}+\cdots \\
& y_{4}(x)=1+x^{2}+\frac{x^{4}}{2}+\frac{x^{6}}{6}+\frac{x^{8}}{24}+\frac{47 x^{10}}{5775}+\cdots \\
& y_{5}(x)=1+x^{2}+\frac{x^{4}}{2}+\frac{x^{6}}{6}+\frac{x^{8}}{24}+\frac{x^{10}}{120}+\frac{2489 x^{12}}{1801800}+\cdots \\
& y_{6}(x)=1+x^{2}+\frac{x^{4}}{2}+\frac{x^{6}}{6}+\frac{x^{8}}{24}+\frac{x^{10}}{120}+\frac{x^{12}}{720}+\frac{12499 x^{14}}{63063000}+\cdots \\
& y_{7}(x)=1+x^{2}+\frac{x^{4}}{2}+\frac{x^{6}}{6}+\frac{x^{8}}{24}+\frac{x^{10}}{120}+\frac{x^{12}}{720}+\frac{x^{14}}{5040}+\frac{3323 x^{16}}{134008875}+\cdots
\end{aligned}
$$

Since noise term vanish in limit which gives the exact solution of (33)

$$
y(x)=e^{x^{2}} .
$$

\subsection{Example 6}

Consider the Lane-Emden type problem (6) with $\beta>2$,

$$
y^{\prime \prime}+\frac{8}{x} y+x y=x^{5}-x^{4}+44 x^{2}-30 x
$$

with conditions

$$
y(0)=0, \quad y(2)=8,
$$

the correctional functional for (39) is,

$$
y_{n+1}(x)=y_{n}(x)+\int_{0}^{x} \lambda(\eta)\left(y_{n}^{\prime \prime}(\eta)+\frac{8}{\eta} y_{n}^{\prime}(\eta)-\eta y_{n}(\eta)-\eta^{5}+\eta^{4}-44 \eta^{2}+30 \eta\right) d \eta,(41)
$$

where $\lambda(\eta)=\frac{\eta\left(\eta^{\alpha-1}-x^{\alpha-1}\right)}{(\alpha-1) x^{\alpha-1}}$ as defined in above (16), considering the given initial value, we can select $y_{0}(x)=h x$, where $h$ being any arbitrary constant. 
Using this selection in (41), we obtain the following successive approximation.

$$
\begin{aligned}
& y_{0}(x)=h x \\
& y_{1}(x)=-x^{3}+x^{4}-\frac{h x^{4}}{44}-\frac{x^{6}}{78}+\frac{x^{7}}{98} \\
& y_{2}(x)=-x^{3}+x^{4}+\frac{h x^{7}}{4312}+\frac{x^{9}}{11232}-\frac{x^{10}}{16660} \\
& y_{3}(x)=-x^{3}+x^{4}-\frac{h x^{10}}{733040}-\frac{x^{12}}{2560890}+\frac{x^{13}}{4331600} \\
& y_{4}(x)=-x^{3}+x^{4}-\frac{h x^{13}}{190590400}-\frac{x^{15}}{845095680}-\frac{x^{16}}{1594028800} \\
& y_{5}(x)=-x^{3}+x^{4}-\frac{h x^{16}}{70137267200}-\frac{x^{18}}{380293056000}-\frac{x^{19}}{787450227200}
\end{aligned}
$$

Imposing the boundary condition $y(2)=8$ in all approximations $y_{0}, y_{1}, y_{2}, y_{3}$, $y_{4}, y_{5}, \ldots$ and solving for every value of $h$, we obtain the following sequences for values of $h$.

$$
4,1.33543,0.534976,0.208072,0.0544208,-0.025167, \ldots .
$$

Sequence shows that it converges to zero. Substituting $h=0$ in (42),

$$
\begin{aligned}
& y_{0}(x)=0 \\
& y_{1}(x)=-x^{3}+x^{4}-\frac{x^{6}}{78}+\frac{x^{7}}{98} \\
& y_{2}(x)=-x^{3}+x^{4}+\frac{x^{9}}{11232}-\frac{x^{10}}{16660} \\
& y_{3}(x)=-x^{3}+x^{4}-\frac{x^{12}}{2560890}+\frac{x^{13}}{4331600} \\
& y_{4}(x)=-x^{3}+x^{4}-\frac{x^{15}}{845095680}-\frac{x^{16}}{1594028800}
\end{aligned}
$$




$$
y_{5}(x)=-x^{3}+x^{4}-\frac{x^{18}}{380293056000}-\frac{x^{19}}{787450227200}
$$

Since noise term vanish in limit which gives the exact solution of (37)

$$
y(x)=-x^{3}+x^{4} .
$$

\section{Conclusion}

In this paper, we applied He's VIM to attain an approximate-exact solution of the singular Lane-Emden type equations. This method is applied to both initial value problems and boundary value problems. As this technique is more efficient and reliable but the difficulty of these type problem is due to the existence of singularity at $x=0$.

Our major work is to examine the singular point of Lane-Emden type problems with initial value problems as well as boundary value problems with different Lagrange multipliers. These Lagrange multipliers for each case are different. We demonstrate six distinct examples some of them are linear, nonlinear, homogenous and non-homogenous. Finally, we conclude that the proposed technique is well suit for numerical and analytic solutions.

\section{References}

[1] S. Chandrasekhar, An Introduction to the Study of Stellar Structure, Vol. 2, Courier Corporation, 1957.

[2] A. K. Nasab, A. K1lıçman, Z. P. Atabakan and W. Leong, A numerical approach for solving singular nonlinear Lane-Emden type equations arising in astrophysics, New Astronomy 34 (2015), 178-186. https://doi.org/10.1016/j.newast.2014.06.008

[3] H. T. Davis, Introduction to Nonlinear Differential and Integral Equations, Courier Corporation, 1962.

[4] K. Boubaker and R. A. Van Gorder, Application of the BPES to Lane-Emden equations governing polytropic and isothermal gas spheres, New Astronomy 17(6) (2012), 565-569. https://doi.org/10.1016/j.newast.2012.02.003

[5] R. Krivec and V. Mandelzweig, Numerical investigation of quasilinearization method in quantum mechanics, Computer Physics Communications 138(1) (2001), 69-79. https://doi.org/10.1016/S0010-4655(01)00191-6 
[6] A. Ghorbani and M. Bakherad, A variational iteration method for solving non-linear Lane-Emden problems, New Astronomy 54 (2017), 1-6. https://doi.org/10.1016/j.newast.2016.12.004

[7] Y. Q. Hasan and L. M. Zhu, Solving singular initial value problems in the second-order ordinary differential equations, Journal of Applied Sciences 7(17) (2007), 2505-2508. https://doi.org/10.3923/jas.2007.2505.2508

[8] Y. Q. Hasan and L. M. Zhu, Modified Adomian decomposition method for singular initial value problems in the second-order ordinary differential equations, Surv. Math. Appl. 3 (2008), 183-193.

[9] P. Sharma, Solving singular initial value problems of Emden-Fowler and Lane-Emden type, International Journal of Applied Mathematics and Computation 1(4) (2009), 206212.

[10] N. Shawagfeh, Nonperturbative approximate solution for Lane-Emden equation, J. Math. Phys. 34(9) (1993), 4364-4369. https://doi.org/10.1063/1.530005

[11] A.-M. Wazwaz, A new algorithm for solving differential equations of Lane-Emden type, Appl. Math. Comput. 118(2-3) (2001), 287-310. https://doi.org/10.1016/S0096-3003(99)00223-4

[12] A.-M. Wazwaz, The numerical solution of special fourth-order boundary value problems by the modified decomposition method, Int. J. Comput. Math. 79(3) (2002), 345-356. https://doi.org/10.1080/00207160211928

[13] A.-M. Wazwaz, The modified decomposition method for analytic treatment of differential equations, Appl. Math. Comput. 173(1) (2006), 165-176. https://doi.org/10.1016/j.amc.2005.02.048

[14] G. Adomian, Solution of physical problems by decomposition, Comput. Math. Appl. 27(9-10) (1994), 145-154. https://doi.org/10.1016/0898-1221(94)90132-5

[15] J.-H. He, Variational approach to the Lane-Emden equation, Appl. Math. Comput. 143(23) (2003), 539-541. https://doi.org/10.1016/S0096-3003(02)00382-X

[16] S. Liao, A new analytic algorithm of Lane-Emden type equations, Appl. Math. Comput. 142(1) (2003), 1-16. https://doi.org/10.1016/S0096-3003(02)00943-8

[17] M. Chowdhury and I. Hashim, Solutions of a class of singular second-order IVPs by homotopy-perturbation method, Phys. Lett. A 365(5-6) (2007), 439- 447. https://doi.org/10.1016/j.physleta.2007.02.002 
[18] J. Ramos, Series approach to the Lane-Emden equation and comparison with the homotopy perturbation method, Chaos Solitons Fractals 38(2) (2008), 400-408. https://doi.org/10.1016/j.chaos.2006.11.018

[19] M. Dehghan and F. Shakeri, Approximate solution of a differential equation arising in astrophysics using the variational iteration method, New Astronomy 13(1) (2008), 53-59. https://doi.org/10.1016/j.newast.2007.06.012

[20] H. Goenner and P. Havas, Exact solutions of the generalized Lane-Emden equation, $J$. Math. Phys. 41(10) (2000), 7029-7042. https://doi.org/10.1063/1.1308076

[21] B. Batiha, M. S. M. Noorani and I. Hashim, Numerical solution of sine-Gordon equation by variational iteration method, Phys. Lett. A 370(5-6) (2007), 437-440. https://doi.org/10.1016/j.physleta.2007.05.087

[22] A. Ali and K. Raslan, Variational iteration method for solving biharmonic equations, Phys. Lett. A 370(5-6) (2007), 441-448. https://doi.org/10.1016/j.physleta.2007.05.115

[23] J. He, A variational iteration approach to nonlinear problems and its applications, Mech. Appl. 20(1) (1998), 30-31.

[24] M. Nadeem, F. Li, H. Ahmad, Hes variational iteration method for solving nonhomogeneous cauchy euler differential equations, Nonlinear Sci. Lett. A Math. Phys. Mech. 9(3) (2018), 231-237.

[25] J.-H. He, The variational iteration method for eighth-order initial-boundary value problems, Phys. Scr. 76(6) (2007), 680-682. https://doi.org/10.1088/0031-8949/76/6/016

[26] H. Ahmad, Variational iteration method with an auxiliary parameter for solving differential equations of the fifth order, Nonlinear Sci. Lett. A 9(1) (2018), 27-35.

[27] A.-M. Wazwaz, A reliable treatment of singular Emden-Fowler initial value problems and boundary value problems, Appl. Math. Comput. 217(24) (2011), 10387-10395. https://doi.org/10.1016/j.amc.2011.04.084

[28] H. Ahmad, Variational iteration algorithm-I with an auxiliary parameter for solving Fokker-Planck equation, Earthline J. Math. Sci. 2(1) (2019), 29-37.

https://doi.org/10.34198/ejms.2119.2937 